\title{
Impacto de los derechos de propiedad intelectual sobre la innovación empresarial en Colombia: evidencia a nivel de firma
}

Cómo citar este artículo / To reference this article / Comment citer cet article / Para citar este artigo:

Quintero-Peña, J. W.; Mendoza-Lozano, F. A. (2020). Impacto de los derechos de propiedad intelectual sobre la innovación empresarial en Colombia: evidencia a nivel de firma. Revista Escuela de Administración de Negocios, (89), 131-154.

DOI: https://doi.org/10.21158/01208160.n89.2020.2847

\section{Resumen}

El presente documento examina los efectos de los derechos de propiedad intelectual (DPI) sobre la innovación empresarial, en conformidad con los factores asociados a la innovación. Con base en la Encuesta Empresarial del Banco Mundial (EEBM) para el 2010 y el 2017, la innovación se describe en la clasificación del Manual de Oslo y los DPI según los acuerdos relativos a la propiedad intelectual. En este trabajo se estima un modelo probit con el fin de analizar los efectos de los DPI sobre la innovación en Colombia; se encuentra un efecto positivo y cercano al $10 \%$ de los DPI sobre los distintos tipos de innovación, excepto para las innovaciones en proceso. Por su parte, el efecto de las patentes en las innovaciones en todos los casos es positivo y significativo. Finalmente, a fin de tratar los problemas de sesgo de selección y como método adicional, se utiliza el método de pareo propensity score matching, en el cual el grupo de tratamiento corresponde a las empresas que tienen patentes o cualquier tipo de registro de propiedad intelectual. La muestra se compone de un total de 1935 empresas. Los resultados de los efectos son positivos, especialmente para las empresas que tienen patentes o registros de propiedad intelectual siete años atrás. En conclusión, la evidencia rechaza la hipótesis de Arrow en el caso de Colombia, en el sentido de que las empresas que ya tienen un poder de monopolio generado por los DPI siguen innovando en el tiempo.

Palabras clave: propiedad intelectual; derechos de propiedad intelectual; innovación; innovación empresarial; patentes; registros de propiedad intelectual.

\footnotetext{
Economista de la Universidad del Tolima y Magister en Economía Aplicada del Colegio de la Frontera Norte- Tijuana- México. ORCID: https://orcid.org/0000-0002-6172-0453

2 Ingeniero Industrial y Magister en ciencias económicas de la Universidad Nacional de Colombia. Doctor en Pensamiento Complejo de la Multiversidad Mundo Real Edgar Morin. ORCID: https://orcid.org/0000-0001-5087-4476
} 


\title{
Impact of intellectual property rights on business innovation in Colombia: evidence at firm level
}

\begin{abstract}
This document examines the effects of the Intellectual Property Rights (IPR) on business innovation in accordance with the factors associated with innovation. Based on the World Bank's Enterprise Survey (ES) for 2010 and 2017, innovation is described in the classification of the Oslo Manual and in the IPRs according to the agreements related to intellectual property. In this paper, we estimated a probit model in order to analyze the effects of the IPRs on the innovation in Colombia; resulting on a positive and close to $10 \%$ effect of IPRs on the different types of innovation, except for innovations in progress. The effect of patents on innovations is, in all cases, positive and significant. Finally, in order to deal with selection bias problems and as an additional method, we used the propensity score matching method, with a treatment group corresponding to firms that have patents or any type of intellectual property registration. The sample comprises a total of 1935 firms. The results of the effects are positive, especially for firms that have patents or intellectual property registrations established seven years ago. In conclusion, the evidence rejects Arrow's hypothesis in the case of Colombia, in the sense that firms that already have monopoly power generated by IPRs continue to innovate over time.
\end{abstract}

Keywords: intellectual property; intellectual property rights; innovation; business innovation; patents; intellectual property registrations.

\section{Impactos dos direitos de propriedade intelectual sobre a inovação empresarial na Colômbia: evidências empresariais}

\section{Resumo}

Este documento examina os efeitos dos direitos de propriedade intelectual (DPI) na inovação empresarial, de acordo com os fatores associados à inovação. Com base na Enquete Empresarial do Banco Mundial (EEBM) de 2010 e 2017, a inovação está descrita na classificação do Manual de Oslo e dos DPI segundo acordos relacionados à propriedade intelectual. Neste trabalho, um modelo probit é estimado para analisar os efeitos dos DPI sobre a inovação na Colômbia. Há um efeito positivo de cerca de $10 \%$ dos DPI sobre os diferentes tipos de inovação, exceto para inovações em processo. Por sua vez, o efeito das patentes nas inovações em todos os casos é positivo e significativo. Por fim, para tratar problemas de viés de seleção e como método adicional, é utilizado o método de propensity score matching, em que o grupo de tratamento corresponde às empresas que possuem patentes ou qualquer tipo de registro de propriedade intelectual. A amostra é composta por um total de 1.935 empresas. Os resultados dos efeitos são positivos, principalmente para empresas que possuem patentes ou registros de propriedade intelectual há sete anos. Em conclusão, as evidências rejeitam a hipótese de Arrow no caso da Colômbia, no sentido de que as empresas que já têm um poder de monopólio gerado pelos DPI continuem inovando ao longo do tempo.

Palavras-chave: propriedade intelectual; direitos de propriedade intelectual; inovação; inovação empresarial; patentes; registros de propriedade intelectual. 


\title{
Impact des droits de propriété intellectuelle sur l'innovation \\ commerciale en Colombie: données probantes au niveau de l'entreprise
}

\begin{abstract}
Résumé
Ce document analyse les effets des droits de propriété intellectuelle (DPI) sur l'innovation des entreprises en fonction de facteurs associés à l'innovation. Sur la base de l'enquête entrepreneuriale de la Banque Mondiale (EEBM) sur la période 2010 - 2017, l'innovation est présentée dans la classification du Manuel d'Oslo et des DPI conformément aux accords relatifs à la propriété intellectuelle. Un modèle probit sera estimé afin d'analyser les effets des DPI sur l'innovation en Colombie, où un effet positif de près de $10 \%$ du DPI impacte les différents types d'innovation, à l'exception des innovations en cours. D'un autre côté, l'impact des brevets sur les innovations est positif et significatif. Enfin, pour prendre en compte les problématiques de biais de sélection, nous utiliserons une méthodologie d'appariement du résultat de propension dans laquelle le groupe traité correspond aux entreprises détenant des brevets ou registres de propriété intellectuelle. L'échantillon se compose d'un total de 1935 entreprises. Les résultats des effets des DPI sont positifs, en particulier pour les entreprises possédant des brevets ou ayant enregistré des DPI lors des sept années d'enquête. Finalement, les faits et résultats de l'investigation rejettent l'hypothèse d'Arrow pour la Colombie dans la mesure où les entreprises ayant un certain monopole du fait des DPI continuent par ailleurs leur processus d'innovation.
\end{abstract}

Mots-clés: propriété intellectuelle; droits de propriété intellectuelle; innovation; innovation commerciale; brevets; registres de propriété intellectuelle. 


\section{Introducción}

$\mathrm{E}^{1}$ objetivo del presente trabajo es analizar el impacto de los derechos de propiedad intelectual (DPI) sobre la innovación empresarial en Colombia, mediante datos a nivel de firma. Según Álvarez y García (2012), en la economía mundial la innovación es reconocida como uno de los factores clave del crecimiento económico y la competitividad. Los estudios de Prescott (1998) argumentan que el cambio técnico explica el crecimiento en un 80 $\%$ en los países desarrollados y en un $40 \%$ en los países subdesarrollados. Por otro lado, el enfoque evolucionista sostiene que la adaptación al cambio tecnológico es un asunto de supervivencia entre empresas que se depredan entre sí (Mulder, De Groot y Hofkes, 2001).

Por tanto, existe gran interés académico por comprender los factores que afectan la innovación en los países en desarrollo, tomando como referencia la situación en los países desarrollados. Es relevante analizar, con base en evidencia robusta, los factores que impulsan o dificultan la actividad innovadora en el ámbito empresarial. En este propósito se debe tener en cuenta la influencia del contexto de la innovación: infraestructura, condicionantes institucionales $y$, particularmente, los DPI que se analizan en este trabajo.

Los factores relacionados con la innovación son diversos. Los estudios realizados destacan el proceso de innovación como un continuo aprendizaje en el que las empresas se nutren no solo de su propia experiencia, sino también de una gran variedad de fuentes externas, entre las que se encuentran los consumidores, las universidades, los consultores, los proveedores, los centros de investigación e, incluso, otras empresas competidoras (Arora y Gambardella, 1990). En este contexto, la innovación constituye un proceso prolongado de acumulación de conocimiento a través de las actividades propias de $\mathrm{I}+\mathrm{D}$ y de la interacción con el ambiente. Así, los
DPI representan un reto para los sistemas jurídicos, lo cual se evidencia en una amplia diversidad de reglamentaciones en cada país (WIPO, 2004).

Este trabajo se propone responder dos preguntas: a) ¿cuál es el impacto de los DPI en la innovación empresarial en Colombia?, y b) ¿cómo se distribuyen los impactos de los DPI en la innovación por innovaciones según la clasificación del Manual de Oslo? Es decir que, se busca comprender las implicaciones teóricas y la evidencia cuantitativa del impacto de los DPI sobre la innovación en Colombia a nivel de firma, pues, como lo argumentan ForeroPineda, Laureiro-Martinez y Marín (2011), las firmas son heterogéneas, no solo en términos de su tamaño, sino también en sus capacidades tecnológicas.

Por ende, deben existir diferencias en el comportamiento empresarial innovador $y$ en la productividad (Cohen, 2010; Dosi y Teece, 1998). En el caso colombiano, según la Encuesta Empresarial del Banco Mundial (EEBM), las empresas grandes tienden a ejercer, mayoritariamente, los DPI respecto a las medianas y pequeñas, aunque no presentan diferencias significativas en el número de años de educación de los trabajadores.

La hipótesis de esta investigación está abierta a la búsqueda de evidencia que compruebe un efecto negativo en la propensión a innovar como consecuencia de ejercer los DPI que garantizan posiciones de monopolio, y que a su vez generan una zona de confort, hasta que aparezca un nuevo bien innovador sustituto (Aghion y Howitt, 1990); esta conjetura también se conoce como la Ley de Arrow.

Tal como lo ilustra Williams (2016), si bien es cierto que los DPI propician el trabajo de investigación con inversión privada, un excesivo costo de las patentes puede detener las innovaciones posteriores que se derivan de esos registros. En esa misma línea, el trabajo de Sweet y Eterovic-Maggio (2015) encuentra 
que el efecto de los DPI sobre la innovación no se puede generalizar a cualquier economía, puesto que depende de las condiciones institucionales de cada país. Por ende, la política pública sobre la protección de la propiedad intelectual debe mediar en la tensión que se genera entre la necesidad de proteger la inversión riesgosa de quienes apuestan por la investigación aplicada, los efectos sobre el bienestar social del uso de bienes monopolizados y la continuidad de la inversión en investigación.

Esta tensión está bien descrita para el caso de la investigación biomédica en el trabajo de Heller y Eisenberg (1998): en un paralelo a la «tragedia de los comunes» que ilustra las consecuencias del uso irresponsable de bienes públicos, los DPI tienen el potencial de generar una «tragedia de anticomunes» en la que las invenciones que pueden generar un gran bienestar social entran en desuso por la condición de exclusión y no rivalidad causada por protecciones legales rígidas.

A fin de validar la hipótesis de un efecto negativo en la propensión a innovar como consecuencia de ejercer los DPI, en este trabajo se implementaron dos estrategias empíricas: modelos probabilísticos — probit-, y el método de pareo propensity score matching. Los datos provienen de la EEBM. La muestra se compone de 1935 observaciones válidas y se encuentran resultados en contra de la hipótesis planteada. Es decir, los DPI tienen efecto positivo sobre la innovación empresarial.

Dado que son muy pocos los estudios que utilizan microdatos para analizar las relaciones entre innovación y DPI, este trabajo está en sintonía con las exposición de Maskus (2015), quien hace un llamado a realizar investigaciones de los efectos de los DPI sobre la innovación empresarial con datos a nivel de firma. Consideramos que esta investigación puede ser útil en la formulación y la evaluación de políticas públicas orientadas al mejoramiento de los niveles de innovación en las firmas. También se dejarán explicitas algunas líneas de trabajo que motivan futuras investigaciones. Este estudio cubre aspectos inherentes a la organización, lo cual es un rasgo distintivo del trabajo, dado que la mayoría de los estudios previos se realizaron con variables macroeconómicas.

Este artículo se compone de seis secciones, de las cuales la presente introducción es la primera. La segunda sección muestra la revisión de la literatura sobre los DPI en relación con la innovación empresarial. La tercera presenta la metodología con estadísticas descriptivas de la encuesta. La cuarta está dedicada a exponer los resultados; la quinta sección presenta la discusión de resultados y, por último, la sexta esboza algunas conclusiones.

\section{Revisión de la literatura: innovación y propiedad intelectual}

\begin{abstract}
A partir de los tratados relativos a la propiedad intelectual (TRIPS), en las ciencias sociales ha crecido sustancialmente el interés por estudiar los DPI y su relación con variables de interés económico tales como la innovación, el crecimiento económico, la inversión extranjera, la productividad, los salarios, el desempleo, etc. En particular, la relación entre los DPI y la innovación ha sido objeto de estudio,
\end{abstract}

principalmente desde la implementación de los TRIPS. Así, por ejemplo, Boldrin y Levine (2002) analizan esta relación y concluyen que persisten las diferencias de opinión -entre los economistas y quienes formulan las políticas públicas- acerca del papel exacto que juega la propiedad intelectual (PI) sobre la innovación. 
Por una parte, en teoría, los DPI se consideran absolutamente necesarios, «para alentar el esfuerzo intelectual creativo en aras del interés público»; por otra, se considera que los DPI obstaculizan la competencia, lo que les otorga un papel negativo frente a la innovación. En esta misma línea se encuentra Moser (2012), quien presenta un conjunto único de datos históricos —más de 8000 innovaciones británicas y estadounidenses en ferias mundiales entre 1851 y 1915 - a fin de explorar la relación entre patentes e innovaciones. Los datos indican que la mayoría de las innovaciones - $89 \%$ de las exhibidas por los británicos en 1851 - no fue patentada, pues los inventores eran más propensos a innovar en industrias en las que las innovaciones son fáciles de copiar, gracias a la ingeniería inversa, lo cual hace que el secreto guardado por la patente sea ineficaz.

Por su parte, Maskus (2015) hace una revisión de los efectos de la implementación de los DPI en las últimas décadas y concluye que la implementación de los DPI ha tenido como resultado la estandarización y armonización de las regulaciones, aun cuando algunos países mantengan cierta flexibilidad. También muestra que los cambios en los costos de los DPI han sido mayores con respecto a los cambios registrados en el sector transporte y el sector de comercio internacional.

Park (2007) analiza las distinciones que se derivan del modelo norte-sur y concluye que la innovación ocurre típicamente en el norte y las tecnologías se difunden hacia el sur, bien sea a través de la imitación por agentes del sur o a través de mecanismos de transferencia de tecnología tales como licencias o inversión extranjera directa. En este sentido, la biodiversidad y el conocimiento étnico son otros de los temas en la agenda de los DPI en los países en desarrollo. La biodiversidad en países de Centroamérica y Suramérica es particularmente llamativa para muchas universidades y multinacionales de países desarrollados interesadas en investigar y explotar especies de plantas nativas con propiedades importantes.

Concluye, además, que la tasa de innovación puede variar positiva o negativamente con DPI más fuertes, dependiendo de su nivel inicial (Park, 2007). Esto da lugar a relaciones no monótonas ni lineales entre los DPI y la innovación. Sugiere, en particular, una relación de $U$ invertida entre la innovación y las patentes.

Para Dixon y Greenhalgh (2002) existe una tensión entre la política de competencia y las políticas relativas a los DPI. La tensión se presenta cuando las autoridades restringen el poder de los monopolistas en el mercado, con el objetivo de mejorar el bienestar de los consumidores. En las décadas recientes, en el marco de los TRIPS, las políticas de regulación de los DPI se han implementado en muchos países, en atención, principalmente, a casos de gran visibilidad en los medios de comunicación, tales como el sector farmacéutico y el informático.

Para el caso de Colombia existe evidencia de la relación negativa entre innovación y DPI, en particular en ciertas industrias. Rossi et al. (2013) encuentran que la relación entre la proporción de la inversión en innovación destinada a investigación básica y aplicada de la firma y su decisión de patentar es, en contra de lo esperado, negativa. También comprueban que la relación entre el número de innovaciones de productos influye positivamente en la decisión de patentar. 


\section{Metodología}

\subsection{Datos}

T a información sobre los DPI y la innovación en Colombia proviene de la EEBM para los años 2010 y 2017. El estudio se encuentra limitado a las empresas que respondieron el cuestionario sobre los DPI en el 2010 e innovación en el 2017. La muestra se compone de un total de 1935 empresas.

La encuesta provee temáticas amplias; entre estas se encuentran las capacidades tecnológicas de las empresas en diversos sectores de la economía colombiana, las cuales permiten analizar la innovación y la implementación de los DPI. Es posible identificar las innovaciones según el Manual de Oslo, porque se incluyen preguntas asociadas a: a) productos o servicios, b) mercado, c) procesos, y d) cambios organizacionales.

En el 2017, los tipos de innovación se reducen a la primera y tercera innovación. Además, como proxy de la innovación, se puede identificar las empresas que han realizado inversiones en investigación y desarrollo. A fin de identificar el uso de DPI, se consideran las siguientes dos preguntas: a) ¿en los últimos tres años la empresa presentó una patente, un registro de marca $\mathrm{y}$ derechos de autor para un producto o proceso?, y b) ¿se tiene registrada una patente?

\subsection{Estadísticas descriptivas}

En la tabla 1 se presentan las descriptivas básicas de las empresas que participaron en la EEBM. En lo referente a ejercer los DPI en el 2010, el 68,2 \% de las empresas reportó no tener ningún registro en los últimos tres años. Más del $50 \%$ de las empresas innovan e invierten en investigación y desarrollo.

$\mathrm{Al}$ parecer, ejercer los DPI se relaciona con mayores niveles de innovación en todas las clasificaciones del Manual de Oslo. Se destaca el incremento en las innovaciones en proceso, ya que empresas sin registros de DPI innovan en un 47,7 \%, mientras que si ejercen los DPI la innovación es del 65,1 \%. Las empresas que ejercen los DPI, en promedio, invierten un $26 \%$ más en investigación y desarrollo respecto a empresas que no los tienen. En promedio la edad de las empresas es 24 años; se aprecia que empresas con más años en el mercado presentan mayor número de DPI.

Solo el $23 \%$ de las empresas colaboran con otras organizaciones en el propósito de innovar, mientras que con el sector público la colaboración es del el 14 $\%$. Se aprecia que a mayor tamaño de la empresa la colaboración para innovar aumenta: empresas grandes colaboran un $10 \%$ más respecto a las pequeñas.

Las empresas con registros de patente cooperan en un 5,3\% adicional con otras organizaciones a fin de realizar actividades de innovación, con respecto a las que no tienen patentes. No se aprecian grandes diferencias en lo referente a la colaboración con el sector público para innovar, incluso por el tamaño de las empresas (véase el Anexo).

Las empresas con registro de patentes colaboran en 25 puntos porcentuales adicionales con el sector público, con relación a las que no tienen este tipo de registros. El hecho de tener cualquier tipo de registro de DPI, incluida la patente, implica un $8 \%$ adicional en la proporción de empresas que colaboran con el sector público (véase el Anexo).

Es resaltable que las empresas que tienen registro de DPI y patentes se relacionan con un mayor número de certificaciones de calidad; en ambos casos, la diferencia es de 20 puntos porcentuales respecto a las que no tienen DPI o patente.

La participación de capital extranjero en las empresas se incrementa en promedio en 6 puntos porcentuales cuando se ejercen los DPI y las patentes. Por otro lado, esta participación aumenta con el tamaño de las empresas, puesto que la diferencia entre pequeñas y grandes es muy notable, pasando del 1,8 \% al 21,5 $\%$. El promedio del número de años de educación de los trabajadores es del 10,6 \%. En las empresas que ejercen DPI y patentes la educación promedio de 
los trabajadores es mayor en tan solo seis meses. Lo anterior refleja las bajas capacidades de innovación en la planta de personal de las empresas colombianas (véase el Anexo).

Los sectores, según la taxonomía de Pavitt, que presentan mayores registros de DPI son: basado en la ciencia, dominadas por escala, dominadas por proveedores, sectores intensivos en información y proveedores especializados, respectivamente (véase el Anexo).
Se evidencia que los registros se dan en empresas en las que su producto o servicio tiene pocos competidores, específicamente menos de cinco competidores, lo cual se relaciona con el poder de monopolio. A su vez, las empresas con registros de DPI se enfrentan a un alto nivel de competencia informal, lo que podría explicar, en parte, las altas tasas de innovación en las empresas colombianas.

Tabla 1. Estadísticas descriptivas

\begin{tabular}{|l|c|c|c|c|c|}
\hline \multicolumn{1}{|c|}{ Variable } & Obs. & Media & Desvest. & Mín. & Máx. \\
\hline Inversiones en DPI & 705 & 0,318 & 0,466 & 0 & 1 \\
\hline Registros en DPI & 705 & 0,277 & .448 & 0 & 1 \\
\hline Innovación 1 & 705 & 0,614 & 0,487 & 0 & 1 \\
\hline Innovación 2 & 433 & 0,536 & 0,499 & 0 & 1 \\
\hline Innovación 3 & 705 & 0,574 & 0,495 & 0 & 1 \\
\hline Innovación 4 & 405 & 0,526 & 0,5 & 0 & 1 \\
\hline Inversión en I+D & 705 & 0,573 & 0,495 & 0 & 1 \\
\hline Edad & 942 & 23,797 & 17147 & 2 & 105 \\
\hline Colaboración privada & 705 & 0,23 & 0,421 & 0 & 1 \\
\hline Colaboración pública & 705 & 0,142 & 0,349 & 0 & 1 \\
\hline Mercado externo & 705 & 0,04 & 0,195 & 0 & 1 \\
\hline Más de cinco competidores & 942 & 0,022 & 0,148 & 0 & 1 \\
\hline Competencia informal & 942 & 0,749 & 0,434 & 0 & 1 \\
\hline Participación de capital extranjero & 942 & 0,099 & 0,298 & 0 & 1 \\
\hline Mujeres en la junta directiva & 942 & 0,416 & 0,493 & 0 & 1 \\
\hline Experiencia top manager & 940 & 23,846 & 11,8 & 1 & 66 \\
\hline Certificado de calidad & 942 & 0,296 & 0,457 & 0 & 1 \\
\hline Acceso al crédito & 942 & 0,732 & 0,443 & 0 & 1 \\
\hline Número de empleados & 942 & 129,172 & 574421 & 1 & 15533 \\
\hline Educación de los trabajadores & 689 & 10,614 & 1991 & 5 & 20 \\
\hline
\end{tabular}

Fuente. Elaboración propia con base a EEBM.

\subsection{Especificación empírica modelo probit}

La presente sección muestra la especificación utilizada para examinar las variables que afectan la probabilidad de innovar de las empresas colombianas. Se supone la presencia de una variable latente $y^{*}$, dada por:

$$
\boldsymbol{y}_{\mathrm{i}}^{*}=\boldsymbol{X}_{i} \boldsymbol{\beta}+\boldsymbol{u}_{i}(\mathbf{1})
$$

Donde $\boldsymbol{X}_{i}$ es un vector kxl de la variable control para la empresa $i, \boldsymbol{\beta}$ es un vector $\mathrm{kx} 1$ de coeficientes, $\mathrm{y} \boldsymbol{u}_{i}$ es error normalmente distribuido para cada variable $\boldsymbol{X}_{\boldsymbol{i}}$. Sin embargo, en lugar de $\boldsymbol{y}_{\boldsymbol{i}}^{*}$, se considerará una variable binaria $\boldsymbol{y} \boldsymbol{i}$, la cual indica el signo de $\boldsymbol{y}_{\boldsymbol{i}}^{*}$ observado. Es decir, 


$$
y i=\left\{\begin{array}{l}
1 \text { si } y_{i}^{*}>0 \\
0 \text { si } y_{i}^{*} \leq 0
\end{array}\right\}(2)
$$

Donde $\boldsymbol{y} \boldsymbol{i}$ toma el valor de 1 si la empresa ha innovado en los últimos tres años. Así, si la variable latente $\boldsymbol{y}_{\mathrm{i}}^{*}$ excede cero, el evento ocurre. De lo contrario, el evento no ocurre. En el modelo probabilístico probit la probabilidad de que la variable yi tome el valor de uno, condicionado a un vector de variables $\boldsymbol{X}_{i}$, está dado por:

$$
\operatorname{Pr}=\left(y_{i}=\frac{1}{x i}\right)=\phi\left(X_{j}^{\prime} \beta\right)
$$

Donde $\phi$ representa la distribución estándar de probabilidad normal, y $\beta$ es el coeficiente asociado con $x_{i}$. Los coeficientes del modelo probit pueden interpretarse como un pequeño cambio o un infinitesimal en las covariables. No obstante, un análisis más intuitivo de los coeficientes requiere calcular los efectos marginales, los cuales se pueden representar de la siguiente manera:

$$
\frac{\partial \operatorname{Pr}\left(y_{i}=1 / X_{j i}\right)}{\partial X}=\frac{\partial \phi\left(X_{j}^{\prime} \beta\right)}{\partial X_{j i}}
$$

Específicamente, se propone el siguiente modelo:

$$
Y_{i}=\gamma(D P I)+\beta X_{i}+\phi W_{i}+u_{i}
$$

En la tabla 2 se especifica el vector de variables $\boldsymbol{Y}_{i}$, la variable dependiente, que corresponde a las asociadas con la definición de innovación del Manual de Oslo. D1 toma el valor de 1 si las empresas encuestadas responden que si tiene una innovación en producto. Así mismo, D2 indaga sobre innovación en mercado; D3, en proceso y D4 organizacional. También se realiza con respecto a la decisión de invertir en $\mathrm{I}+\mathrm{D}$, como proxy de innovación. Los DPI, igualmente, se muestran en la tabla 2.

El vector $X_{i}$ representa las variables a nivel empresarial que afectan la innovación, las cuales son las siguientes: orientación al exterior, número de competidores - uno o más—, tamaño de la empresa - pequeña, mediana, grande-, edad, experiencia del top manager, certificaciones de calidad, acceso al mercado financiero, educación de los trabajadores, cooperación con otras empresas, apoyo de fondos públicos, participación de la directiva mujer en la junta y presencia de capital extranjero en la empresa. También se discrimina por la taxonomía de Pavitt (1985) y la ampliación de Tidd y Bessant (2001), quien agregó sectores intensivos en información sector servicios- (véase el Anexo).

Tabla 2. Variables del modelo

\begin{tabular}{|c|l|c|}
\hline \multicolumn{2}{|c|}{ Variables asociadas a los DPI } & Fspecificación \\
\hline Variable & \multicolumn{1}{|c|}{ Fuente } \\
\hline Patente & Tener registrada una patente. & EEBM \\
\hline Ejercer los DPI-EDPI & Si en los últimos tres años la empresa registró: patentes, marcas, derechos de autor. & EEBM \\
\hline \multicolumn{2}{|c|}{ Variables dependiente-innovación — dicótomas, toma el valor de 1, si se cumple la condición- } \\
\hline Innovación 1 & Si la empresa en los últimos tres años introdujo una innovación en producto. & EEBM \\
\hline Innovación 2 & Si la empresa en los últimos tres años introdujo una innovación en mercado. & EEBM \\
\hline Innovación 3 & Si la empresa en los últimos tres años introdujo una innovación en proceso & EEBM \\
\hline Innovación 4 & Si la empresa en los últimos tres años introdujo una innovación organizacional. & EEBM \\
\hline Inversión en I+D & Si la empresa en los últimos tres años ha realizado inversiones en I+D. & EEBM \\
\hline
\end{tabular}

Fuente. Elaboración propia con base a la EEBM. 


\section{Resultados}

T a tabla 3 presenta los efectos marginales del modelo probit, en los que las variables dependientes se Urepresentan en las columnas. Las cuatro primeras corresponden a las categorías de innovación según el Manual de Oslo, tal como se referenció antes. La quinta columna representa la variable dependiente « gasto en investigación y desarrollo».

Tabla 3. Efectos marginales. Probabilidad de innovar 2010

\begin{tabular}{|c|c|c|c|c|c|}
\hline & $-1-$ & $-2-$ & $-3-$ & $-4-$ & $-5-$ \\
\hline Variables & Producto & Mercado & Proceso & Organizacional & Gasto id \\
\hline \multirow[t]{2}{*}{ Ejercer los DPI } & $0,133^{* * *}$ & $0,158^{* * *}$ & 0,0475 & $0,123^{* *}$ & $0,197^{* * *}$ \\
\hline & $-0,0439-$ & $-0,0543-$ & $-0,0466-$ & $-0,0591-$ & $-0,0441-$ \\
\hline \multirow[t]{2}{*}{ Más de cinco competidores } & $-0,0599$ & $-0,0607$ & $-0,0684^{*}$ & 0,0354 & $-0,117^{* * *}$ \\
\hline & $-0,0410-$ & $-0,0545-$ & $-0,0415-$ & $-0,0559-$ & $-0,0421-$ \\
\hline \multirow[t]{2}{*}{ Empresa mediana } & 0,0226 & 0,0215 & 0,0463 & $-0,0751$ & $-0,0397$ \\
\hline & $-0,0477-$ & $-0,0650-$ & $-0,0484-$ & $-0,0673-$ & $-0,0502-$ \\
\hline \multirow[t]{2}{*}{ Empresa grande } & 0,0766 & 0,0436 & $0,112^{* *}$ & $-0,0193$ & 0,0184 \\
\hline & $-0,0542-$ & $-0,0754-$ & $-0,0547-$ & $-0,0786-$ & $-0,0575-$ \\
\hline \multirow[t]{2}{*}{ Edad de la empresa } & $-0,00156$ & 0,000200 & $-0,000317$ & $-0,00250$ & 0,000119 \\
\hline & $-0,00129-$ & $-0,00163-$ & $-0,00126-$ & $-0,00158-$ & $-0,00138-$ \\
\hline \multirow[t]{2}{*}{ Experiencia del top manager } & 0,00147 & $-0,00201$ & $-0,00345^{* *}$ & 0,000799 & $-0,000879$ \\
\hline & $-0,00177-$ & $-0,00232-$ & $-0,00174-$ & $-0,00229-$ & $-0,00183-$ \\
\hline \multirow[t]{2}{*}{ Certificación de calidad } & 0,0139 & $-0,0385$ & 0,0229 & $-0,0237$ & $0,139^{* * *}$ \\
\hline & $-0,0483-$ & $-0,0628-$ & $-0,0504-$ & $-0,0657-$ & $-0,0490-$ \\
\hline \multirow[t]{2}{*}{ Competencia con informales } & $0,120^{* *}$ & 0,0785 & 0,0816 & 0,0809 & $0,0970^{*}$ \\
\hline & $-0,0501-$ & $-0,0653-$ & $-0,0498-$ & $-0,0696-$ & $-0,0528-$ \\
\hline \multirow[t]{2}{*}{ Acceso al crédito } & 0,0431 & $-0,0744$ & 0,0173 & $-0,0344$ & 0,0587 \\
\hline & $-0,0470-$ & $-0,0621-$ & $-0,0468-$ & $-0,0654-$ & $-0,0491-$ \\
\hline \multirow[t]{2}{*}{ Años de educación trabajadores } & 0,0106 & $-0,0137$ & 0,000244 & $-0,00234$ & 0,0135 \\
\hline & $-0,0102-$ & $-0,0139-$ & $-0,0103-$ & $-0,0139-$ & $-0,0104-$ \\
\hline \multirow[t]{2}{*}{ Colaboración privada } & $0,134^{* * *}$ & $0,142^{* *}$ & $0,192^{* * *}$ & $0,157^{* * *}$ & $0,257^{* * *}$ \\
\hline & $-0,0464-$ & $-0,0593-$ & $-0,0456-$ & $-0,0604-$ & $-0,0434-$ \\
\hline \multirow[t]{3}{*}{ Colaboración pública } & $0,143^{* * *}$ & $-0,122^{*}$ & 0,0736 & 0,0134 & 0,0895 \\
\hline & $-0,0539-$ & $-0,0712-$ & $-0,0592-$ & $-0,0755-$ & $-0,0613-$ \\
\hline & $-0,126-$ & $-0,157-$ & $-0,141-$ & $-0,182-$ & $-0,140-$ \\
\hline \multirow[t]{2}{*}{ Participación mujer en JD } & $0,107^{* * *}$ & 0,0559 & $0,0701^{*}$ & $-0,0350$ & 0,0270 \\
\hline & $-0,0389-$ & $-0,0519-$ & $-0,0406-$ & $-0,0556-$ & $-0,0418-$ \\
\hline \multirow[t]{2}{*}{ Capital extranjero } & $0,207^{* * *}$ & 0,0850 & $-0,00149$ & $-0,00457$ & 0,0526 \\
\hline & $-0,0584-$ & $-0,0848-$ & $-0,0772-$ & $-0,0967-$ & $-0,0827-$ \\
\hline Efecto industria & SI & SI & SI & SI & SI \\
\hline Observaciones & 658 & 409 & 658 & 381 & 658 \\
\hline
\end{tabular}

Errores estándar robustos. Las estimaciones presentan clúster sector según la taxonomía de Pavitt. ${ }^{* * *} \mathrm{p}<0,01,{ }^{* *} \mathrm{p}<0,05,{ }^{*} \mathrm{p}<0,1$

Fuente. Elaboración propia. 
En el inicio de esta investigación se consideró el efecto de los DPI sobre la innovación. Como hipótesis, se propuso un escenario en el que los DPI inhiben la innovación debido al poder de monopolio que se genera. Sin embargo, luego del procedimiento empírico, se encontró lo contrario; es decir, empresas que implementan los DPI tienen incentivos para seguir innovando. En el 2010 (véase la Tabla 3, columna 1), se aprecia que es un $13 \%$ más probable innovar en producto o servicio cuando se ejercen los DPI respecto a empresas que no lo hacen. Por otro lado, el efecto de una patente es positivo en un $8 \%$ adicional en la probabilidad de innovar, respecto a empresas que no tienen ese registro (véase el Anexo). Las variables control que tienen un efecto significativo sobre la innovación en producto o servicio son: la competencia informal, apoyo de otras empresas para innovar, participación del sector público, la participación de la mujer en la junta directiva de la empresa y participación de capital extranjero. La competencia informal influye de manera positiva en la innovación, lo que puede explicarse por las capacidades de innovación de las empresas formales $y$, por otro lado, como estrategia de supervivencia empresarial, teniendo en cuenta que la formalidad implica costos adicionales.

La cooperación con otras empresas para innovar tiene un efecto positivo y es significativo solo para empresas pequeñas y medianas. Por otra parte, la participación del sector público en procesos de innovación tiene un efecto positivo sobre la innovación en producto o servicio, sin embargo, solo es significativa para empresas grandes, dado que que el hecho de tener apoyos del sector público aumenta la probabilidad de innovar en un $23 \%$ (véase el Anexo). Lo anterior puede explicarse por el hecho de que empresas grandes tienen las capacidades y los requisitos para participar en convocatorias públicas relacionadas con investigación y desarrollo. La presencia de mujeres en la junta directiva tiene un efecto positivo sobre la innovación en producto; sin embargo, es significativo solo en empresas grandes.

Tabla 4. Efectos marginales. Probabilidad de innovar en producto 2017 dada una innovación en proceso en el 2010

\begin{tabular}{|l|c|c|c|c|}
\hline \multirow{4}{*}{ Variables } & $-\mathbf{- 1}$ & $\mathbf{- 2 -}$ & $\mathbf{- 3 -}$ & $\mathbf{- 4 -}$ \\
\hline \multirow{2}{*}{ Innova proceso -t-7- } & $\begin{array}{c}\text { Innovación } \\
\text { producto }\end{array}$ & $\begin{array}{c}\text { Innovación } \\
\text { producto }\end{array}$ & $\begin{array}{c}\text { Innovación } \\
\text { producto }\end{array}$ & $\begin{array}{c}\text { Innovación } \\
\text { producto }\end{array}$ \\
\hline \multirow{2}{*}{ Ejercer los DPI -t-7- } & & $0,215^{* * *}$ & & \\
\hline \multirow{3}{*}{$>5$ competidores } & $0,136^{* * *}$ & $0,133^{* * *}$ & $0,0880^{*}$ & \\
\cline { 2 - 5 } & $-0,0431-$ & $-0,0437-$ & $-0,0516-$ & \\
\hline \multirow{2}{*}{ Mediana } & $-0,0631$ & $-0,0508$ & $-0,0412$ & $-0,0566$ \\
\hline \multirow{2}{*}{ Grande } & $-0,0413-$ & $-0,0420-$ & $-0,0483-$ & $-0,0415-$ \\
\hline \multirow{2}{*}{ Edad } & 0,0353 & 0,0261 & 0,0182 & 0,0426 \\
\hline \multirow{2}{*}{ Experiencia top manager } & $-0,0476-$ & $-0,0483-$ & $-0,0588-$ & $-0,0473-$ \\
\hline \multirow{2}{*}{ Certificaciones } & 0,0865 & 0,0652 & 0,0521 & $0,101^{*}$ \\
\cline { 2 - 6 } & $-0,0543-$ & $-0,0558-$ & $-0,0668-$ & $-0,0533-$ \\
\hline
\end{tabular}




\begin{tabular}{|c|c|c|c|c|}
\hline \multirow{2}{*}{ Competencia informal } & $0,110^{* *}$ & $0,0962^{*}$ & 0,0467 & $0,118^{* *}$ \\
\hline & $-0,0507-$ & $-0,0511-$ & $-0,0621-$ & $-0,0509-$ \\
\hline \multirow{2}{*}{ Acceso al crédito } & 0,0336 & 0,0308 & $-0,0305$ & 0,0425 \\
\hline & $-00471-$ & $-0,0470-$ & $-0,0561-$ & $-0,0468-$ \\
\hline \multirow{2}{*}{ Educación de los trabajadores } & 0,0107 & 0,0110 & 0,00778 & 0,0134 \\
\hline & $-0,0103-$ & $-0,0104-$ & $-0,0124-$ & $-0,0102-$ \\
\hline \multirow{2}{*}{ Colaboración privada } & $0,130^{* * *}$ & $0,0936^{*}$ & 0,0415 & $0,132^{* * *}$ \\
\hline & $-0,0467-$ & $-0,0496-$ & $-0,0540-$ & $-0,0465-$ \\
\hline \multirow{2}{*}{ Colaboración pública } & $0,154^{* * *}$ & $0,143^{* *}$ & $0,128^{* *}$ & $0,165^{* * *}$ \\
\hline & $-0,0541-$ & $-0,0561-$ & $-0,0574-$ & $-0,0532-$ \\
\hline \multirow{2}{*}{ Mujeres en la junta directiva } & $0,0970^{* *}$ & $0,0838^{* *}$ & 0,0528 & $0,109^{* * *}$ \\
\hline & $-0,0393-$ & $-0,0398-$ & $-0,0477-$ & $-0,0392-$ \\
\hline \multirow{2}{*}{ Participación capital extranjero } & $0,211^{* * *}$ & $0,214^{* * *}$ & $0,173^{* * *}$ & $0,207^{* * *}$ \\
\hline & $-0,0571-$ & $-0,0578-$ & $-0,0559-$ & $-0,0581-$ \\
\hline \multirow{2}{*}{ Innova organizacional 2010} & & & $0,102^{* *}$ & \\
\hline & & & $-0,0466-$ & \\
\hline \multirow{2}{*}{ Patente - $\mathrm{t}-7-$} & & & & $00923^{* *}$ \\
\hline & & & & $-0,0424-$ \\
\hline Efecto industria & SI & SI & SI & SI \\
\hline Observaciones & 651 & 651 & 376 & 651 \\
\hline
\end{tabular}

Errores estándar robustos. Las estimaciones presentan clúster sector según la taxonomía de Pavitt. ${ }^{* *} \mathrm{p}<0,01,{ }^{* *} \mathrm{p}<0,05,{ }^{*} \mathrm{p}<0,1$

Fuente. Elaboración propia.

Tabla 5. Efectos marginales. Probabilidad de innovar en producto 2017 dada una innovación en producto 2010

\begin{tabular}{|c|c|c|c|c|c|}
\hline & $-1-$ & $-2-$ & $-3-$ & $-4-$ & $-5-$ \\
\hline Variables & $\begin{array}{c}\text { Innovación } \\
\text { proceso }\end{array}$ & $\begin{array}{c}\text { Innovación } \\
\text { proceso }\end{array}$ & $\begin{array}{c}\text { Innovación } \\
\text { proceso }\end{array}$ & $\begin{array}{c}\text { Innovación } \\
\text { proceso }\end{array}$ & $\begin{array}{c}\text { Innovación } \\
\text { proceso }\end{array}$ \\
\hline \multirow{2}{*}{ Innova producto $-\mathrm{t}-7-$} & $0,226^{* * *}$ & & & $0,223^{* * *}$ & \\
\hline & $-0,0414-$ & & & $-0,0413-$ & \\
\hline \multirow{2}{*}{ Ejercer los DPI — $\mathrm{t}-7$ - } & 0,00895 & $-0,0340$ & & & \\
\hline & $-0,0478-$ & $-0,0546-$ & & & \\
\hline \multirow{2}{*}{$>5$ competidores } & $-0,0557$ & $-0,0308$ & $-0,0674$ & $-0,0556$ & $-0,0323$ \\
\hline & $-0,0427-$ & $-0,0507-$ & $-0,0419-$ & $-0,0426-$ & $-0,0505-$ \\
\hline \multirow{2}{*}{ Mediana } & 0,0472 & 0,00148 & 0,0558 & 0,0489 & $-4,88 \mathrm{e}-05$ \\
\hline & $-0,0488-$ & $-0,0595-$ & $-0,0482-$ & $-0,0488-$ & $-0,0594-$ \\
\hline \multirow{2}{*}{ Grande } & $0,106^{*}$ & 0,0742 & $0,124^{* *}$ & $0,109^{* *}$ & 0,0693 \\
\hline & $-0,0552-$ & $-0,0669-$ & $-0,0539-$ & $-0,0550-$ & $-0,0664-$ \\
\hline \multirow{2}{*}{ Edad } & $-0,000409$ & 0,000116 & $-0,000915$ & $-0,000598$ & $8,66 \mathrm{e}-05$ \\
\hline & $-0,00128-$ & $-0,00150-$ & $-0,00127-$ & $-0,00128-$ & $-0,00152-$ \\
\hline \multirow{2}{*}{ Experiencia top manager } & $-0,00385^{* *}$ & $-0,00281$ & $-0,00336^{*}$ & $-0,00383^{* *}$ & $-0,00283$ \\
\hline & $-0,00179-$ & $-0,00217-$ & $-0,00177-$ & $-0,00179-$ & $-0,00217$ \\
\hline \multirow{2}{*}{ Certificaciones } & 0,0177 & $-0,0523$ & 0,0153 & 0,0113 & $-0,0549$ \\
\hline & $-0,0521-$ & $-0,0591-$ & $-0,0510-$ & $-0,0522-$ & $-0,0590-$ \\
\hline
\end{tabular}


Impacto de los derechos de propiedad intelectual sobre la innovación empresarial en Colombia:

\begin{tabular}{|l|c|c|c|c|c|}
\hline \multirow{2}{*}{ Competencia informal } & 0,0588 & 0,0316 & $0,0832^{*}$ & 0,0586 & 0,0300 \\
\cline { 2 - 6 } & $-0,0508-$ & $-0,0614-$ & $-0,0502-$ & $-0,0507-$ & $-0,0613-$ \\
\hline \multirow{3}{*}{ Acceso al crédito } & 0,00323 & $-0,0511$ & 0,0135 & 0,00387 & $-0,0546$ \\
\cline { 2 - 6 } & $-0,0469-$ & $-0,0586-$ & $-0,0470-$ & $-0,0469-$ & $-0,0583-$ \\
\hline \multirow{2}{*}{ Educación de los trabajadores } & $-0,00500$ & $-0,00340$ & $-0,00206$ & $-0,00536$ & $-0,00428$ \\
\cline { 2 - 6 } & $-0,0106-$ & $-0,0134-$ & $-0,0105-$ & $-0,0106-$ & $-0,0132-$ \\
\hline \multirow{2}{*}{ Colaboración privada } & $0,176^{* * *}$ & $0,111^{* *}$ & $0,198^{* * *}$ & $0,177^{* * *}$ & $0,112^{* *}$ \\
\hline \multirow{2}{*}{ Colaboración pública } & $-0,0480-$ & $-0,0530-$ & $-0,0459-$ & $-0,0478-$ & $-0,0529-$ \\
\hline \multirow{2}{*}{ Mujeres en la JD } & 0,0475 & 0,0914 & 0,0803 & 0,0512 & 0,0920 \\
\cline { 2 - 6 } & $-0,0633-$ & $-0,0603-$ & $-0,0603-$ & $-0,0631-$ & $-0,0602-$ \\
\hline \multirow{2}{*}{ Capital extranjero } & 0,0520 & 0,0233 & $0,0804^{* *}$ & 0,0586 & 0,0226 \\
\hline \multirow{2}{*}{ Innovacion organizacional 2010 } & $-0,0416-$ & $-0,0493-$ & $-0,0408-$ & $-0,0416-$ & $-0,0496-$ \\
\hline \multirow{2}{*}{ Patente - t-7- } & $-0,0416$ & $-0,0495$ & $-3,26 \mathrm{e}-05$ & $-0,0444$ & $-0,0502$ \\
\hline & $-0,0801-$ & $-0,0849-$ & $-0,0763-$ & $-0,0796-$ & $-0,0848-$ \\
\hline Observaciones & & $0,233^{* * *}$ & & & $0,229^{* * *}$ \\
\hline
\end{tabular}

Errores estándar robustos. Las estimaciones presentan clúster sector según la taxonomía de Pavitt. ${ }^{* *} \mathrm{p}<0,01,{ }^{* *} \mathrm{p}<0,05,{ }^{*} \mathrm{p}<0,1$

Fuente. Elaboración propia.

Con respecto a la innovación en mercado, los resultados del 2010 reflejan que se mantienen los efectos positivos de las mismas variables que la innovación en producto excepto la participación de capital extranjero, mujeres en la junta directiva y competencia informal. También es claro que ejercer los DPI tiene un efecto positivo. En contraste, es revelador el efecto negativo de participar en una red con instituciones públicas (véase la Tabla 3), lo que refleja una posible carencia de las políticas públicas orientadas a fomentar la innovación en el componente de comercialización.

Los efectos de los DPI por tamaño de empresa son solo significativos para empresas pequeñas y medianas. Las empresas pequeñas que tienen registros de DPI tienen una mayor probabilidad de innovar en un $24 \%$ respecto a empresas del mismo tamaño que no tiene registros. Sin embargo, para el caso de las patentes se evidencia mayor propensión a innovar en empresas grandes como resultados de una patente, y el efecto no es significativo para empresas pequeñas (véase el Anexo).
Las innovaciones en proceso se ven impactadas positivamente al existir la cooperación con otras empresas. En este caso, el efecto de la práctica de trabajo en red es mayor al efecto sobre las dos primeras formas de innovación (véase la Tabla 3). De nuevo, la participación de mujeres en las juntas directivas tiene efectos positivos en este tipo de innovación. Si la empresa tiene más de cinco competidores, la probabilidad de innovar en procesos tiene un efecto negativo en la innovación (véase la Tabla 3). Este es el único tipo de innovación que no tiene efectos positivos y significativos derivado de los registros de DPI, sin embargo, la tenencia de patente si tiene efectos significativos, aunque son ligeramente menores respecto a los otros tipos de innovación.

Las innovaciones organizacionales se ven afectadas de forma positiva por los registros de DPI; es un $12 \%$ más probable innovar organizacionalmente al comparar con empresas que no tenga registros. La mayoría de las variables de control no son significativas, excepto la cooperación para innovar con otras empresas (véase la Tabla 3 ). 
En la columna 5 de la tabla de efectos marginales por patente (véase el Anexo), se muestran los efectos en la decisión de invertir en investigación y desarrollo, que es una variable ampliamente usada como proxy de innovación empresarial; además, en la base de datos se puede verificar el monto de la inversión. Los resultados de los coeficientes son de mayor magnitud a los desagregados en los diferentes tipos de innovaciones. Tener registros en DPI aumenta la probabilidad de realizar inversiones en cerca de un $20 \%$.

Además, competir con más de cinco empresas disminuye la probabilidad en un $11 \%$ de invertir respecto a empresas que tienen cierto poder de mercado - pocos competidores-. El resultado vislumbra que las empresas caracterizadas por tener poder de monopolio tienen mayor propensión a innovar. Resultados similares se evidencian con la patente, mas la diferencia radica en que la participación del sector público es significativa y su efecto es en sentido directo con una probabilidad del $10 \%$.

\subsection{Efecto de los DPI obtenidos en el 2010 sobre las innovaciones en el 2017}

Con información de innovaciones en producto o servicio reportados en el 2017 se muestra que haber tenido DPI en el 2010 genera efectos positivos en la innovación del segundo periodo. Igualmente, si una empresa en el 2010 tenía una patente, la innovación es más probable en cerca de un $10 \%$ en el 2017. Lo anterior indica que los DPI generan incentivos a la innovación. También es un $10 \%$ más probable que una empresa para el 2017 innove en producto, si en el 2010 realizó una innovación en proceso (véase la Tabla 4).

El hecho de que la empresa siete años atrás haya realizado una innovación en proceso hace un $21 \%$ más probable que innove en producto en el 2017, mientras que si la innovación fue organizacional el efecto es la mitad respecto a una innovación en proceso. Igualmente, empresas que reportaron registros de patentes siete años atrás tienen una probabilidad de innovar del $9 \%$ adicional respecto a las que no la tienen; este porcentaje es ligeramente mayor al que se encontró en el 2010, lo que podría explicarse por el hecho de que la patente ya se está madurando. Por tanto, funciona como un incentivo a seguir innovando.

Innovar en procesos en el 2017 es más probable para empresas que realizaron innovaciones en producto siete años atrás; este es un resultado esperado puesto que los cambios en los productos o servicios generan disrupciones en los procesos de las empresas. La cooperación con otras empresas es significativa, mientras la cooperación con el sector público no lo es a menos que se haya realizado una innovación organizacional en el 2010. El efecto de la innovación organizacional del 2010 sobre la de procesos en el 2017 es similar a la de producto (véase la Tabla 5).

\subsection{Resultados del método de pareo - propensity score matching-. Impacto de los DPI sobre la innovación empresarial}

Con base en las estimaciones de un modelo probit se establece la propensión a patentar y ejercer los DPI. Luego se generan los grupos de tratamiento y de control. El primero corresponde a empresas que patentaron o implementaron algún DPI, mientras que el grupo de control corresponde a empresas que no presentan ningún registro de DPI. Cada empresa del grupo de tratamiento se empareja con una del grupo de control.

Lo anterior se realiza mediante los siguientes métodos: los diez vecinos más cercanos, kernel y el método de estratificación. A fin de asegurar un buen balance, el pareo es restringido a observaciones con soporte común. Mediante el método de los diez vecinos más cercanos, se ve cómo las firmas que ejercieron los DPI en el 2010 tienen una propensión a realizar innovaciones en mercado, y el $9 \%$ mayor con respecto a las empresas que no tienen ningún tipo de registro. El efecto es significativo en magnitud, dado que el $53 \%$ de las empresas realizan innovaciones en mercado.

Las patentes tienen impactos significativos sobre las innovaciones en general, no así sobre las innovaciones organizacionales. El hecho de tener una patente 
en el 2010 aumenta la probabilidad de innovación en producto en cerca de un $5 \%$, con respecto a las empresas del grupo de control. Impactos similares se pueden apreciar para las innovaciones en proceso. Efectos mayores se pueden evidenciar también para el caso de las innovaciones en mercado, puesto que en este caso la probabilidad de innovar en el grupo de tratamiento es un $11 \%$ mayor con respecto al grupo de control. Lo anterior puede explicarse por el hecho de que, una vez se tiene la patente, es necesario generar estrategias de mercado en el proceso de apropiabilidad.

Finalmente, la propensión a invertir en investigación y desarrollo es cercana al $10 \%$. Sin embargo, los resultados anteriores se ven limitados por el periodo de tiempo. Por tanto, la tabla A1 del anexo muestra el efecto de las empresas que registraron patentes o cualquier otro registro de DPI en el 2010 sobre los resultados de la innovación en el 2017. Se pueden apreciar efectos más grandes al compararlos con los anteriores, ya que si una empresa tiene registros de DPI en el 2010, la probabilidad de innovar en producto en el 2017 es $18 \%$ mayor con respecto al grupo de control. Para el caso de las innovaciones en proceso no se encontraron efectos significativos.

Cuando se toman los efectos de las patentes del 2010 del grupo de tratamiento sobre la innovación del 2017 se encuentra que la probabilidad de innovar en producto es un $10 \%$ mayor con respecto al grupo de control. Ahora, el efecto de las patentes del 2010 sobre la innovación en procesos en el 2017 es positiva, resultado muy similar al anterior tanto en dirección como en magnitud. Los resultados anteriores se pueden ver en el anexo.

\section{Discusión}

$\mathrm{E}_{8}^{1}$ presente estudio ha explorado si los DPI generan efectos en la innovación de las empresas colombianas. Con base a la Encuesta empresarial del Banco Mundial en sus rondas del 2010 y el 2017, respectivamente, el análisis confirma que los DPI tienen un efecto positivo sobre innovaciones posteriores. Este resultado contradice la hipótesis de Arrow, según la cual empresas que tienen registros de DPI dejan de innovar.

Sin embargo, los resultados van en línea con estudios anteriores; por ejemplo, Forero-Pineda et al. (2011) señalan que las empresas pequeñas con patentes son menos innovadoras en términos de nuevos productos, resultado que va en sintonía con los hallazgos en el presente estudio, porque empresas pequeñas son menos propensas a innovar en producto respecto a empresas grandes, dado que el efecto de la patente es positivo.

Además, cuando se analizan efectos por tamaño se aprecia que el efecto de los registros de DPI no es significativo en productos para pequeñas empresas, solo el efecto es positivo cuando las pequeñas empresas innovan en mercado. Resultados contrarios en comparación con los estudios de Rossi et al. (2013), quienes en relación con el presente estudio encuentran que la relación entre la proporción de la inversión en innovación destinada a investigación básica y aplicada de la firma y su decisión de patentar es, en contra de lo esperado, negativa y significativa a un nivel del $5 \%$.

Pero, si bien el estudio anterior se realizó para la industria manufacturera colombiana, los resultados podrían variar debido a que en se incluyeron controles de subsectores específicos tales como alimentos y bebidas, químicos, cauchos y plásticos, y muebles. Mientras el presente estudio utiliza la clasificación de Pavitt, encontrando que la inversión en investigación y desarrollo es más probable si el establecimiento tiene una patente, en particular las empresas de los sectores intensivos en escala es más probable que innoven respecto a los proveedores dominantes. 
Rossi et al. (2013) también prueban la hipótesis según la cual la orientación de la firma hacia la innovación en productos influye positivamente en su decisión de patentar; no tienen en cuenta los problemas de causalidad reversa entre la innovación y la patente. En el presente estudio la decisión de innovar en producto o servicio es un 9,2 \% más probable, resultado similar cuando se compara con el grupo tratamiento, es decir, en el 2017 es cerca de un $10 \%$ más probable que innoven respecto a empresas que no tienen patentes — grupo control—y un $18 \%$ más probable cuando se compara con cualquier registro de DPI en el 2010.
En general, los resultados se aproximan a los hallazgos de Yang, Huang y Lin (2014), ya que encuentran que los DPI son beneficiosos para fomentar las innovaciones en países de ingresos medios altos desarrollados-, mientras que muestra una influencia diversa en las innovaciones para países en desarrollo en diversas estimaciones. $\mathrm{Al}$ usar datos agregados del Banco Mundial, Colombia es clasificado como un país de ingreso medio alto.

\section{Conclusiones}

$\mathrm{E}_{\mathrm{s}}^{\mathrm{se}}$ te trabajo indagó por el efecto de ejercer los DPI en cuatro categorías de innovación. Las capacidades tecnológicas de las empresas se caracterizaron y fueron explorados con la EEMB para los años 2010 y 2017. Usando el análisis de regresión y los métodos de pareo, se analizó el efecto de los DPI sobre la innovación empresarial en Colombia. Se encontró un efecto positivo, lo cual descarta la hipótesis de una zona de confort que inhibe la innovación como consecuencia de las ventajas de mercado.

La hipótesis se rechaza, en concordancia con otros estudios, como, por ejemplo, el de Sweet y EterovicMaggio (2015), los cuales sostienen que el efecto positivo es posible siempre y cuando se cuente con una estructura institucional fuerte que garantice la protección de los DPI. Por ende, la evidencia motiva futuras investigaciones en torno al funcionamiento de esa institucionalidad en el caso colombiano.

Esta investigación también corrobora el efecto positivo de la participación en redes públicas y privadas en las cuatro formas de innovación que se consideraron en la encuesta. Es resaltable que a la luz de la evidencia se encuentren efectos positivos de la participación de las mujeres en las juntas directivas de las empresas colombianas, en coherencia con hallazgos similares que se derivan de los estudios que ilustran los beneficios de la diversidad en el género sobre la innovación (Ruiz-Jiménez, Fuentes-Fuentes y Ruiz-Arroyo, 2016).

Finalmente, el hallazgo del efecto positivo del cambio organizacional sobre las diferentes formas de innovación son un aporte para los futuros trabajos que se orienten a caracterizar esas transformaciones en el contexto colombiano.

\section{Referencias}

Aghion, P.; Howitt, P. (1990). A model of growth through creative destruction. Cambridge: National Bureau of Economic Research. DOI: https://doi.org/10.3386/w3223

Álvarez, E.; García, W. (2012). Determinantes de la innovación: evidencia en el sector manufacturero de bogotá. Semestre Económico,15(32), 129-160.DOI: https://doi.org/10.22395/ seec.v15n32a5

Arora, A.; Gambardella, A. (1990). Complementarity and external linkages: the strategies of the large firms in biotechnology. The Journal of Industrial Economics, 38(4), 361-379. DOI: https:// doi.org/10.2307/2098345

Boldrin, M.; Levine, D. (2002). The case against intellectual property. American Economic Review, 92(2), 209-212. DOI: https://doi.org/10.1257/000282802320189267 
Cohen, W.M. (2010). Fifty years of empirical studies of innovative activity and performance. En H. Bronwyn; N. Rosenberg (Eds.) Handbook of the Economics of Innovation. (vol. 1, 129213). Amsterdam: Elsevier. DOI: https://doi.org/10.1016/ S0169-7218(10)01004-X

Dixon, P.; Greenhalgh, C. (2002). The economics of intellectual property: a review to identify themes for future research. Oxford: University of Oxford.

Dosi, G.; Teece, D. J. (1998). Organizational competencies and the boundaries of the firm. En C. Longhi; R. Arena (Eds.) Markets and organization. (281-302). Berlín: Springer-Verlag. DOI: https://doi.org/10.1007/978-3-642-72043-7_12

Forero-Pineda, C.; Laureiro-Martinez, D.; Marín, A. (2011). Innovation patterns and intellectual property in SME's of a developing country. Innovar, 21(42). Recuperado de https:// bit.ly/3vcclnH

Heller, M. A.; Eisenberg, R. S. (1998). Can patents deter innovation? The anticommons in biomedical research. Science, 280(5364), 698-701. DOI: https://doi.org/10.1126/ science.280.5364.698

Maskus, K. E. (2015). Intellectual property in a globalizing world: issues for economic research. Asia-Pacific Journal of Accounting \& Economics, 22(3), 231-250. DOI: https://doi.org/10.1080 $/ 16081625.2015 .1057947$

Moser, P. (2012). Innovation without patents: evidence from world's fairs. The Journal of Law and Economics, 55(1), 43-74. DOI: https://doi.org/10.1086/663631

Mulder, P.; De Groot, H. L. F.; Hofkes, M. W. (2001). Economic growth and technological change: a comparison of insights from a neo-classical and an evolutionary perspective. Technological Forecasting and Social Change, 68(2), 151-171. DOI: https://doi.org/10.1016/S0040-1625(00)00078-0

Park, W. G. (2007). Chapter 9 intellectual property rights and international innovation. En K. E. Maskus (Ed.) Intellectual property, growth and trade. (289-327). Bingley: Emerald Group Publishing Limited. DOI: https://doi.org/10.1016/ S1574-8715(07)00009-7
Pavitt, K. (1984). Sectoral patterns of technical change: Towards a taxonomy and a theory. Research Policy, 13(6), 343-373. DOI: https://doi.org/10.1016/0048-7333(84)90018-0

Prescott, E. (1998). Needed: a theory of total factor productivity, International Economic Review, 39(3), 525-551. DOI: https:// doi.org/10.2307/2527389

Rossi, J. L. J.; Barrios-Aguirre, F.; Schmutzler, J.; SánchezManchola, I. D. (2013). Relación entre la estrategia de innovación de la firma y su decisión de patentar: evidencia de empresas pertenecientes al sector manufacturero colombiano. Estudios Gerenciales, 29(128), 313-321. DOI: https://doi. org/10.1016/j.estger.2013.09.005

Ruiz-Jiménez, J.M.; Fuentes-Fuentes, M.; Ruiz-Arroyo, M. (2016). Knowledge Combination Capability and Innovation: The Effects of Gender Diversity on Top Management Teams in Technology-Based Firms. Journal of Business Ethics,135, 503-515. DOI: https://doi.org/10.1007/s10551-014-24627

Sweet, C. M.; Eterovic-Maggio, D. S. (2015). Do stronger intellectual property rights increase innovation? World Development, 66, 665-677. DOI: https://doi.org/10.1016/j. worlddev.2014.08.025

Tidd, J.; Bessant, J. R. (2001). Managing innovation: integrating technological, market and organizational change. Estados Unidos: John Wiley \& Sons.

Williams, H. L. (2016). Intellectual property rights and innovation: evidence from health care markets. Innovation Policy and the Economy, 16(1), 53-87. DOI: https://doi. org/10.1086/684986

WIPO. (2004). WIPO intellectual property handbook: policy, law and use. Ginebra: WIPO. Recuperado de https://bit. ly/2PcbqnU

Yang, C.-H.; Huang, Y.-J.; Lin, H.-Y. (2014). Do stronger intellectual property rights induce more innovations? A Cross-country Analysis. Hitotsubashi Journal of Economics, 55(2), 167-188. 


\section{Anexo}

Tabla A1. Efectos marginales. Probabilidad de innovar al tener patente

\begin{tabular}{|c|c|c|c|c|c|}
\hline & $-1-$ & $-2-$ & $-3-$ & $-4-$ & $-5-$ \\
\hline Variables & Producto & Mercado & Proceso & Organizacional & Gasto_id \\
\hline \multirow{2}{*}{ Patente } & $0,0849^{* *}$ & $0,0975^{*}$ & $0,0792^{*}$ & $0,107^{*}$ & $0,143^{* * *}$ \\
\hline & $-0,0424-$ & $-0,0543-$ & $-0,0437-$ & $-0,0566-$ & $-0,0432-$ \\
\hline \multirow{2}{*}{ Varios competidores } & $-0,0549$ & $-0,0523$ & $-0,0667$ & 0,0446 & $-0,109^{* *}$ \\
\hline & $-0,0412-$ & $-0,0545-$ & $-0,0414-$ & $-0,0558-$ & $-0,0424-$ \\
\hline \multirow{2}{*}{ Mediana } & 0,0318 & 0,0341 & 0,0496 & $-0,0698$ & $-0,0250$ \\
\hline & $-0,0474-$ & $-0,0651-$ & $-0,0483-$ & $-0,0671-$ & $-0,0497-$ \\
\hline \multirow{2}{*}{ Grande } & $0,0926^{*}$ & 0,0691 & $0,118^{* *}$ & $-0,00198$ & 0,0446 \\
\hline & $-0,0531-$ & $-0,0744-$ & $-0,0544-$ & $-0,0781-$ & $-0,0572-$ \\
\hline \multirow{2}{*}{ Edad } & $-0,00160$ & 0,000140 & $-0,000471$ & $-0,00264$ & $-8,86 e-05$ \\
\hline & $-0,00129-$ & $-0,00163-$ & $-0,00125-$ & $-0,00161-$ & $-0,00136-$ \\
\hline \multirow{2}{*}{ Experiencia top manager } & 0,00142 & $-0,00207$ & $-0,00340^{*}$ & 0,000712 & $-0,000939$ \\
\hline & $-0,00177-$ & $-0,00232-$ & $-0,00174-$ & $-0,00231-$ & $-0,00183-$ \\
\hline \multirow{2}{*}{ Certificaciones } & 0,0164 & $-0,0269$ & 0,0196 & $-0,0226$ & $0,143^{* * *}$ \\
\hline & $-0,0481-$ & $-0,0624-$ & $-0,0505-$ & $-0,0657-$ & $-0,0486-$ \\
\hline \multirow{2}{*}{ Competencia informal } & $0,128^{* *}$ & 0,0902 & $0,0830^{*}$ & 0,0953 & $0,109^{* *}$ \\
\hline & $-0,0501-$ & $-0,0644-$ & $-0,0496-$ & $-0,0688-$ & $-0,0525-$ \\
\hline \multirow{2}{*}{ Acceso al crédito } & 0,0518 & $-0,0618$ & 0,0207 & $-0,0330$ & 0,0708 \\
\hline & $-0,0467-$ & $-0,0627-$ & $-0,0469-$ & $-0,0655-$ & $-0,0486-$ \\
\hline \multirow{2}{*}{ Educación trabajadores } & 0,0133 & $-0,00977$ & 0,00102 & 0,000901 & $0,0178^{*}$ \\
\hline & $-0,0101-$ & $-0,0139-$ & $-0,0103-$ & $-0,0138-$ & $-0,0103-$ \\
\hline \multirow{2}{*}{ Colaboración privada } & $0,136^{* * *}$ & $0,142^{* *}$ & $0,193^{* * *}$ & $0,158^{* * *}$ & $0,261^{* * *}$ \\
\hline & $-0,0462-$ & $-0,0591-$ & $-0,0455-$ & $-0,0597-$ & $-0,0428-$ \\
\hline \multirow{3}{*}{ Colaboración pública } & $0,154^{* * *}$ & $-0,121^{*}$ & 0,0782 & 0,0262 & $0,103^{*}$ \\
\hline & $-0,0529-$ & $-0,0704-$ & $-0,0589-$ & $-0,0752-$ & $-0,0587-$ \\
\hline & $0,118^{* * *}$ & 0,0699 & $0,0782^{*}$ & $-0,0224$ & 0,0429 \\
\hline \multirow{2}{*}{ Participación de mujeres en la JD } & $-0,0388-$ & $-0,0519-$ & $-0,0406-$ & $-0,0557-$ & $-0,0416-$ \\
\hline & $0,202^{* * *}$ & 0,0821 & $-0,00519$ & $-0,00939$ & 0,0448 \\
\hline Capital extranjero & $-0,0592-$ & $-0,0837-$ & $-0,0768-$ & $-0,0973-$ & $-0,0826-$ \\
\hline Efecto industria & SI & SI & SI & SI & SI \\
\hline Observaciones & 658 & 409 & 658 & 381 & 658 \\
\hline
\end{tabular}

Errores estándar robustos, Las estimaciones presentan clúster sector según la taxonomía de Pavitt, ${ }^{* * *} \mathrm{p}<0,01,{ }^{* *} \mathrm{p}<0,05,{ }^{*} \mathrm{p}<0,1$

Fuente. Elaboración propia. 
Tabla A2. Diferencias entre empresas que tienen registros de DPI

\begin{tabular}{|l|c|c|c|c|}
\hline & \multicolumn{2}{|c|}{ Empresas sin registro de DPI } & \multicolumn{2}{c|}{ Empresas con registro de DPI } \\
\hline Innovación 1 & 510 & 0,563 & 195 & 0,749 \\
\hline Innovación 2 & 287 & 0,477 & 146 & 0,651 \\
\hline Innovación 3 & 510 & 0,547 & 195 & 0,646 \\
\hline Innovación 4 & 279 & 0,498 & 126 & 0,587 \\
\hline Inversión en I+D & 510 & 0,5 & 195 & 0,764 \\
\hline Inversión en I+D(Miles de dólares) & 240 & $1,62 \mathrm{e}+08$ & 136 & $8,17 \mathrm{e}+08$ \\
\hline Edad & 510 & 23,439 & 195 & 29,374 \\
\hline Redes & 510 & 0,206 & 195 & 0,292 \\
\hline Colaboración pública & 510 & 0,12 & 195 & 0,2 \\
\hline Mercado externo & 510 & 0,033 & 195 & 0,056 \\
\hline Más de cinco competidores & 510 & 0,035 & 195 & 0,015 \\
\hline Competencia informal & 510 & 0,757 & 195 & 0,8 \\
\hline Participación de capital extranjero & 510 & 0,084 & 195 & 0,144 \\
\hline Mujeres en la junta directiva & 510 & 0,431 & 195 & 0,426 \\
\hline EDAD1 & 510 & 23,439 & 195 & 29,374 \\
\hline Experiencia top manager & 508 & 24,053 & 195 & 23,749 \\
\hline Certificado de calidad & 510 & 0,263 & 195 & 0,456 \\
\hline Acceso al crédito & 510 & 0,729 & 195 & 0,815 \\
\hline Número de empleados & 510 & 74,378 & 195 & 296,385 \\
\hline Educación de los trabajadores & 499 & 10,465 & 190 & 11,005 \\
\hline
\end{tabular}

Fuente. Elaboración propia con base a EEBM.

Tabla A3. Según la clasificación taxonómica de Pavitt

\begin{tabular}{|l|c|c|c|c|c|c|c|c|c|c|}
\hline & \multicolumn{2}{|c|}{$\begin{array}{c}\text { Sipplier } \\
\text { dominted }\end{array}$} & \multicolumn{2}{c|}{$\begin{array}{c}\text { Scale } \\
\text { intensive }\end{array}$} & \multicolumn{2}{c|}{$\begin{array}{c}\text { Especialized } \\
\text { supplier }\end{array}$} & \multicolumn{2}{c|}{$\begin{array}{c}\text { Sciense } \\
\text { based }\end{array}$} & \multicolumn{2}{c|}{$\begin{array}{c}\text { Information } \\
\text { intensive }\end{array}$} \\
\cline { 2 - 13 } & N Promedio & \multicolumn{2}{|c|}{ N Promedio } & \multicolumn{2}{c|}{ N Promedio } & \multicolumn{2}{c|}{ N Promedio } & \multicolumn{2}{c|}{ N Promedio } \\
\hline Inversiones en DPI & 413 & 0,32 & 3 & 0,333 & 167 & 0,162 & 107 & 0,551 & 15 & 0,333 \\
\hline $\begin{array}{l}\text { Inversiones - miles } \\
\text { de dólares- }\end{array}$ & 118 & $4,29 \mathrm{e}+08$ & 1 & 1000000 & 24 & $5,77 \mathrm{e}+07$ & 55 & $2,90 \mathrm{e}+08$ & 4 & $2,25 \mathrm{e}+07$ \\
\hline Registros en DPI & 413 & 0,278 & 3 & 0,333 & 167 & 0,18 & 107 & 0,43 & 15 & 0,2 \\
\hline Innovación 1 & 413 & 0,62 & 3 & 0,333 & 167 & 0,575 & 107 & 0,654 & 15 & 0,667 \\
\hline Innovación 2 & 256 & 0,531 & 1 & 1 & 96 & 0,573 & 70 & 0,529 & 10 & 0,3 \\
\hline Innovación 3 & 413 & 0,581 & 3 & 0,333 & 167 & 0,587 & 107 & 0,551 & 15 & 0,467 \\
\hline Innovación 4 & 240 & 0,504 & 1 & 0 & 98 & 0,633 & 59 &, 475 & 7 & 0,286 \\
\hline Inversión en I+D & 413 & 0,564 & 3 & 0,333 & 167 & 0,521 & 107 &, 701 & 15 & 0,533 \\
\hline $\begin{array}{l}\text { Inversión en I+D - } \\
\text { miles de dólares- }\end{array}$ & 217 & $3,81 \mathrm{e}+08$ & 0 &, & 81 & $2,44 \mathrm{e}+08$ & 71 & $6,53 \mathrm{e}+08$ & 7 & $1,79 \mathrm{e}+08$ \\
\hline Edad & 431 & 25,276 & 31 & 20,935 & 170 & 21,924 & 117 & 28,667 & 193 & 19,653 \\
\hline Redes & 413 & 0,201 & 3 & 0,333 & 167 & 0,251 & 107 & 0,308 & 15 & 0,2 \\
\hline $\begin{array}{l}\text { Colaboración } \\
\text { pública }\end{array}$ & 413 & 0,145 & 3 & 0 & 167 & 0,138 & 107 & 0,15 & 15 & 0,067 \\
\hline Mercado externo & 413 & 0,053 & 3 & 0 & 167 & 0,03 & 107 & 0 & 15 & 0,067 \\
\hline
\end{tabular}




\begin{tabular}{|l|c|c|c|c|c|c|c|c|c|c|}
\hline Monopolio & 431 & 0,032 & 31 & 0 & 170 & 0,029 & 117 & 0,009 & 193 & 0,005 \\
\hline $\begin{array}{l}\text { Competencia } \\
\text { informal }\end{array}$ & 431 & 0,81 & 31 & 0,516 & 170 & 0,753 & 117 & 0,641 & 193 & 0,715 \\
\hline $\begin{array}{l}\text { Participación de } \\
\text { capital extranjero }\end{array}$ & 431 & 0,079 & 31 & 0,129 & 170 & 0,1 & 117 & 0,214 & 193 & 0,067 \\
\hline $\begin{array}{l}\text { Mujeres en la junta } \\
\text { directiva }\end{array}$ & 431 & 0,464 & 31 & 0,323 & 170 & 0,394 & 117 & 0,333 & 193 & 0,394 \\
\hline EDAD1 & 431 & 25,276 & 31 & 20,935 & 170 & 21,924 & 117 & 28,667 & 193 & 19,653 \\
\hline $\begin{array}{l}\text { Experiencia top } \\
\text { mánager }\end{array}$ & 429 & 23,601 & 31 & 26,645 & 170 & 24,053 & 117 & 24,607 & 193 & 23,295 \\
\hline $\begin{array}{l}\text { Certificado de } \\
\text { calidad }\end{array}$ & 431 & 0,267 & 31 & 0,452 & 170 & 0,347 & 117 & 0,419 & 193 & 0,218 \\
\hline Acceso al crédito & 431 & 0,78 & 31 & 0,645 & 170 & 0,718 & 117 & 0,718 & 193 & 0,663 \\
\hline $\begin{array}{l}\text { Número de } \\
\text { empleados }\end{array}$ & 431 & 156,71 & 31 & 157 & 170 & 74,835 & 117 & 140,47 & 193 & 104,218 \\
\hline $\begin{array}{l}\text { Educación de los } \\
\text { trabajadores }\end{array}$ & 400 & 10,383 & 3 & 10,667 & 166 & 10,735 & 105 & 11,19 & 15 & 11,4 \\
\hline
\end{tabular}

Fuente. Elaboración propia con base a EEBM.

Tabla A4. Estadísticas descriptivas según el tamaño de la empresa

\begin{tabular}{|c|c|c|c|c|c|c|}
\hline \multirow{2}{*}{$\begin{array}{l}\text { Variable } \\
\text { Inversiones en DPI }\end{array}$} & \multicolumn{2}{|c|}{ Pequeña } & \multicolumn{2}{|c|}{ Mediana } & \multicolumn{2}{|c|}{ Grande } \\
\hline & 203 & 0,202 & 266 & 0,312 & 233 & 0,425 \\
\hline Inversiones —-miles de dólares- & 38 & $3,01 \mathrm{e}+07$ & 78 & $1,50 \mathrm{e}+07$ & 85 & $7,73 e+08$ \\
\hline Registros en DPI & 203 & 0,177 & 266 & 0,259 & 233 & ,378 \\
\hline Innovación 1 & 203 & 0,567 & 266 & 0,609 & 233 & ,665 \\
\hline Innovación 2 & 115 & 0,522 & 162 & 0,525 & 155 & ,555 \\
\hline Innovación 3 & 203 & 0,522 & 266 & 0,568 & 233 & ,627 \\
\hline Innovación 4 & 106 & 0,585 & 151 & 0,51 & 146 & ,507 \\
\hline Inversión en I+D & 203 & 0,522 & 266 & 0,538 & 233 & ,665 \\
\hline Inversión en I+D —-miles de dólores_- & 99 & $2,57 e+07$ & 133 & $4,98 \mathrm{e}+07$ & 144 & $9,79 \mathrm{e}+08$ \\
\hline Cooperación con otras empre & 277 & 17,982 & 350 & 22,049 & 312 & 30,965 \\
\hline Redes & 203 & 0,197 & 266 & 0,207 & 233 & 0,288 \\
\hline Colaboración pública & 203 & 0,113 & 266 & 0,154 & 233 & 0,155 \\
\hline Mercado externo & 203 & 0,02 & 266 & 0,038 & 233 & 0,06 \\
\hline Más de 5 competidores & 277 & 0,025 & 350 & 0,034 & 312 & 0,006 \\
\hline Competencia informal & 277 & 0,791 & 350 & 0,783 & 312 & 0,673 \\
\hline Participación de capital extranjero & 277 & 0,018 & 350 & 0,06 & 312 & 0,215 \\
\hline Mujeres en la junta directiva & 277 & 0,484 & 350 & 0,429 & 312 & 34 \\
\hline EDAD1 & 277 & 17,982 & 350 & 22,049 & 312 & 30,965 \\
\hline Experiencia top manager & 276 & 23,301 & 350 & 24,18 & 311 & 24,016 \\
\hline Certificado de calidad & 277 & 0,13 & 350 & 0,263 & 312 & ,484 \\
\hline Acceso al crédito & 277 & 0,635 & 350 & 0,746 & 312 & 804 \\
\hline Número de empleados & 277 & 17,765 & 350 & 45,737 & 312 & 322,718 \\
\hline Educación de los trabajadores & 199 & 10,563 & 264 & 10,322 & 223 & 11,009 \\
\hline
\end{tabular}

Fuente. Elaboración propia con base a EEBM. 
Tabla A5. Efectos marginales por tamaño de empresa y tipo de innovación

\begin{tabular}{|c|c|c|c|c|c|c|}
\hline \multirow[b]{3}{*}{ Varia } & \multicolumn{2}{|c|}{ Producto } & \multicolumn{4}{|c|}{ Mercado } \\
\hline & $-1-$ & $-2-$ & $-3-$ & $-4-$ & $-5-$ & $-6-$ \\
\hline & Pequeña & Mediana & Grandes & Pequeña & Mediana & Grandes \\
\hline \multirow{2}{*}{ Ejercer los DPI } & 0,0386 & 0,0749 & $0,209^{* * *}$ & $0,243^{* *}$ & $0,188^{* *}$ & 0,107 \\
\hline & $-0,106-$ & $-0,0761-$ & $-0,0641-$ & $-0,124-$ & $-0,0915-$ & $-0,0878-$ \\
\hline \multirow{2}{*}{ Varios competidores } & $-0,113$ & $-0,0364$ & $-0,0273$ & $-0,0751$ & $-0,138$ & $-0,00523$ \\
\hline & $-0,0787-$ & $-0,0686-$ & $-0,0714-$ & $-0,108-$ & $-0,0908-$ & $-0,0997-$ \\
\hline \multirow{2}{*}{ Edad } & $-0,00604^{* *}$ & $-0,00129$ & $-0,000659$ & $-0,0101^{* *}$ & 0,00281 & 0,00167 \\
\hline & $-0,00304-$ & $-0,00227-$ & $-0,00188-$ & $-0,00498-$ & $-0,00318-$ & $-0,00222-$ \\
\hline \multirow{2}{*}{ Experiencia manager } & $0,00614^{*}$ & $-0,00148$ & $-4,65 e-05$ & $-0,00266$ & 0,000509 & $-0,000760$ \\
\hline & $-0,00348-$ & $-0,00315-$ & $-0,00297-$ & $-0,00492-$ & $-0,00432-$ & $-0,00387-$ \\
\hline \multirow{2}{*}{ Certificaciones } & $0,182^{*}$ & $-0,0274$ & $-0,0160$ & $-0,128$ & $-0,0422$ & $-0,0358$ \\
\hline & $-0,0942-$ & $-0,0771-$ & $-0,0778-$ & $-0,143-$ & $-0,104-$ & $-0,103-$ \\
\hline \multirow{2}{*}{ Competencia informal } & 0,0589 & 0,121 & $0,168^{* *}$ & 0,0551 & 0,0960 & 0,0572 \\
\hline & $-0,102-$ & $-0,0874-$ & $-0,0826-$ & $-0,141-$ & $-0,117-$ & $-0,109-$ \\
\hline \multirow{2}{*}{ Acceso al crédito } & 0,0299 & 0,0818 & 0,0620 & $-0,0828$ & $-0,00123$ & $-0,149$ \\
\hline & $-0,0793-$ & $-0,0808-$ & $-0,101-$ & $-0,111-$ & $-0,114-$ & $-0,118-$ \\
\hline \multirow{2}{*}{ Promedio educación T } & $-0,0352^{*}$ & $0,0333^{* *}$ & 0,0127 & $-0,00407$ & $-0,0458^{*}$ & $-0,0104$ \\
\hline & $-0,0211-$ & $-0,0158-$ & $-0,0190-$ & $-0,0252-$ & $-0,0244-$ & $-0,0271-$ \\
\hline \multirow{2}{*}{ Colaboración privada } & $0,194^{* *}$ & $0,169^{* *}$ & 0,0828 & 0,145 & $0,238^{* *}$ & 0,0977 \\
\hline & $-0,0918-$ & $-0,0788-$ & $-0,0765-$ & $-0,121-$ & $-0,0997-$ & $-0,102-$ \\
\hline \multirow{3}{*}{ Colaboración pública } & $-0,0322$ & $0,167^{*}$ & $0,237^{* * *}$ & $-0,00965$ & $-0,201^{*}$ & $-0,170$ \\
\hline & $-0,120-$ & $-0,0888-$ & $-0,0647-$ & $-0,159-$ & $-0,112-$ & $-0,121-$ \\
\hline & 0,115 & $0,139^{* *}$ & 0,104 & 0,0173 & 0,0949 & 0,0577 \\
\hline \multirow{2}{*}{ Part mujer } & $-0,0764-$ & $-0,0655-$ & $-0,0661-$ & $-0,103-$ & $-0,0866-$ & $-0,0894-$ \\
\hline & & $0,215^{* *}$ & $0,216^{* * *}$ & 0,261 & 0,142 & 0,0420 \\
\hline Capital extranjero & & $-0,103-$ & $-0,0706-$ & $-0,294-$ & $-0,172-$ & $-0,107-$ \\
\hline Efecto industria & SI & SI & SI & SI & SI & SI \\
\hline Observaciones & 658 & 409 & 658 & 381 & 658 & 138 \\
\hline
\end{tabular}

Robust standard errors in parentheses ${ }^{* * *} \mathrm{p}<0,01,{ }^{* *} \mathrm{p}<0,05,{ }^{*} \mathrm{p}<0,1$

Fuente. Elaboración propia. 
Tabla A6. Efectos marginales por tamaño de empresa y tipo de innovación —continuación-

\begin{tabular}{|c|c|c|c|c|c|c|}
\hline & \multicolumn{2}{|c|}{ Proceso } & \multicolumn{4}{|c|}{ Organizacional } \\
\hline & $-7-$ & $-8-$ & $-9-$ & $-10-$ & $-11-$ & (12) \\
\hline Variables & Pequeña & Mediana & Grandes & Pequeña & Mediana & Grandes \\
\hline \multirow{2}{*}{ Ejercer los DPI } & 0,141 & $-0,0265$ & 0,0368 & 0,122 & 0,108 & 0.133 \\
\hline & $-0,101-$ & $-0,0782-$ & $-0,0730-$ & $-0,120-$ & $-0,107-$ & $(0.0952)$ \\
\hline \multirow{2}{*}{ Varios competidores } & $-0,00533$ & 0,0261 & $-0,178^{* *}$ & 0,0117 & 0,0391 & 0.0816 \\
\hline & $-0,0802-$ & $-0,0692-$ & $-0,0694-$ & $-0,114-$ & $-0,100-$ & $(0.0951)$ \\
\hline \multirow{2}{*}{ Edad } & $-0,00335$ & 0,00139 & 0,000537 & 0,000248 & $-0,00390$ & -0.00266 \\
\hline & $-0,00317-$ & $-0,00230-$ & $-0,00180-$ & $-0,00419-$ & $-0,00290-$ & $(0.00218)$ \\
\hline \multirow{2}{*}{ Experiencia manager } & $-0,00363$ & $-0,00145$ & $-0,00533^{*}$ & $-0,00237$ & 0,00235 & 0.00227 \\
\hline & $-0,00365-$ & $-0,00300-$ & $-0,00295-$ & $-0,00489-$ & $-0,00381-$ & $(0.00420)$ \\
\hline \multirow{2}{*}{ Certificaciones } & 0,0372 & $-0,0323$ & 0,0962 & $-0,0253$ & $-0,0340$ & -0.00381 \\
\hline & $-0,116-$ & $-0,0793-$ & $-0,0847-$ & $-0,155-$ & $-0,111-$ & $(0.107)$ \\
\hline \multirow{2}{*}{ Competencia informal } & 0,00762 & 0,0545 & $0,158^{*}$ & 0,205 & 0,142 & -0.0419 \\
\hline & $-0,0995-$ & $-0,0875-$ & $-0,0828-$ & $-0,157-$ & $-0,126-$ & $(0.110)$ \\
\hline \multirow{2}{*}{ Acceso al crédito } & $-0,0199$ & 0,111 & $-0,0158$ & 0,0141 & 0,00469 & $-0.211^{*}$ \\
\hline & $-0,0786-$ & $-0,0810-$ & $-0,0931-$ & $-0,119-$ & $-0,122-$ & $(0.115)$ \\
\hline \multirow{2}{*}{ Promedio educación $\mathrm{T}$} & $-0,0339$ & $-0,00820$ & $0,0399^{*}$ & 0,000727 & 0,00343 & 0.0151 \\
\hline & $-0,0211-$ & $-0,0170-$ & $-0,0222-$ & $-0,0298-$ & $-0,0219-$ & $(0.0343)$ \\
\hline \multirow{2}{*}{ Colaboración privada } & $0,224^{* *}$ & $0,214^{* * *}$ & $0,220^{* * *}$ & $0,209^{*}$ & 0,145 & 0.104 \\
\hline & $-0,0921-$ & $-0,0767-$ & $-0,0737-$ & $-0,123-$ & $-0,106-$ & $(0.106)$ \\
\hline \multirow{3}{*}{ Colaboración pública } & $0,379^{* * *}$ & 0,0194 & $-0,123$ & 0,0865 & 0,126 & -0.126 \\
\hline & $-0,0809-$ & $-0,0968-$ & $-0,113-$ & $-0,139-$ & $-0,120-$ & $(0.140)$ \\
\hline & $-0,299-$ & $-0,180-$ & $-0,211-$ & & & $(0.199)$ \\
\hline \multirow{2}{*}{ Part mujer } & 0,0376 & 0,108 & 0,0956 & $-0,0527$ & $-0,0666$ & 0.0295 \\
\hline & $-0,0776-$ & $-0,0662-$ & $-0,0713-$ & $-0,109-$ & $-0,0965-$ & $(0.101)$ \\
\hline \multirow{2}{*}{ Capital extranjero } & $-0,235$ & 0,148 & $-0,0779$ & & $-0,0115$ & 0.0308 \\
\hline & $-0,304-$ & $-0,138-$ & $-0,0987-$ & & $-0,202-$ & $(0.119)$ \\
\hline Efecto industria & SI & SI & SI & SI & SI & SI \\
\hline Observaciones & 194 & 253 & 208 & 99 & 143 & 135 \\
\hline
\end{tabular}

Robust standard errors in parentheses ${ }^{* * *} \mathrm{p}<0,01,{ }^{* *} \mathrm{p}<0,05,{ }^{*} \mathrm{p}<0,1$

Fuente. Elaboración propia. 
Tabla A7. Efectos marginales por tamaño de empresa y tipo de innovación —continuación-

\begin{tabular}{|c|c|c|c|}
\hline & \multicolumn{3}{|c|}{ gasto id } \\
\hline & $-13-$ & $-14-$ & $-15-$ \\
\hline Variables & Pequeña & Mediana & Grandes \\
\hline \multirow{2}{*}{ Ejercer los DPI } & $0,221^{* *}$ & 0,0794 & $0,266^{* * *}$ \\
\hline & $-0,0935-$ & $-0,0788-$ & $-0,0622-$ \\
\hline \multirow{2}{*}{ Varios competidores } & $-0,256^{* * *}$ & 0,0128 & $-0,0949-$ \\
\hline & $-0,0778-$ & $-0,0712-$ & $-0,0714-$ \\
\hline \multirow{2}{*}{ Edad } & $-0,00329$ & $-0,00219$ & 0,00285 \\
\hline & $-0,00369-$ & $-0,00243-$ & $-0,00186-$ \\
\hline \multirow{2}{*}{ Experiencia manager } & $-0,00513$ & 0,000667 & 0,000506 \\
\hline & $-0,00374-$ & $-0,00310-$ & $-0,00296-$ \\
\hline \multirow{2}{*}{ Certificaciones } & 0,166 & $0,171^{* *}$ & 0,121 \\
\hline & $-0,110-$ & $-0,0745-$ & $-0,0814-$ \\
\hline \multirow{2}{*}{ Competencia informal } & $-0,0881$ & $0,192^{* *}$ & 0,0962 \\
\hline & $-0,108-$ & $-0,0853-$ & $-0,0814-$ \\
\hline \multirow{2}{*}{ Acceso al crédito } & 0,115 & 0,0357 & $-0,0172$ \\
\hline & $-0,0815-$ & $-0,0832-$ & $-0,0836-$ \\
\hline \multirow{2}{*}{ Promedio educación T } & $-0,0343$ & 0,0253 & $0,0347^{*}$ \\
\hline & $-0,0209-$ & $-0,0165-$ & $-0,0183-$ \\
\hline \multirow{2}{*}{ Colaboración privada } & $0,270^{* * *}$ & $0,252^{* * *}$ & $0,255^{* * *}$ \\
\hline & $-0,0901-$ & $-0,0761-$ & $-0,0627-$ \\
\hline \multirow{3}{*}{ Colaboración pública } & 0,0793 & 0,0257 & $0,153^{*}$ \\
\hline & $-0,122-$ & $-0,0990-$ & $-0,0894-$ \\
\hline & $-0,0186$ & 0,0558 & 0,0903 \\
\hline \multirow{2}{*}{ Part mujer } & $-0,0798-$ & $-0,0680-$ & $-0,0674-$ \\
\hline & & 0,0598 & 0,0229 \\
\hline Capital extranjero & & $-0,159-$ & $-0,0975-$ \\
\hline Efecto industria & SI & SI & SI \\
\hline Observations & 191 & 253 & 208 \\
\hline
\end{tabular}

Robust standard errors in parentheses ${ }^{* * *} \mathrm{p}<0,01,{ }^{* *} \mathrm{p}<0,05,{ }^{*} \mathrm{p}<0,1$

Fuente. Elaboración propia. 